\title{
The associated factors of HBV and/or HCV with HIV co- infection among men who have sex with men (MSM) in the Northern region of Thailand, 2015
}

\author{
*Denpong Wongwichit ${ }^{1}$, chatubhong Singharachai ${ }^{1}$, Sirinan Suwanaporn ${ }^{1}$, Wipob Suttana ${ }^{1}$, \\ Piyanut Poolviwat ${ }^{2}$, Montha Phetsuwan ${ }^{4}$, Warunya Srisang ${ }^{1}$, Penpayom Suntharn ${ }^{3}$, Chatuporn \\ Phantakrasem ${ }^{1}$ \\ ${ }^{1}$ School of Health Sciences, Mae Fah Luang University, Chiang Rai, Thailand \\ ${ }^{2}$ Mae Fah Luang University Hospital, Chiang Rai, Thailand \\ ${ }^{3}$ Division of Vaccine Preventable Diseases, Department of Disease Control, Ministry of Public Health, Thailand \\ ${ }^{4}$ Northern College, Muang District, Tak province, Thailand \\ ${ }^{*}$ Corresponding Author's E-mail; Denpong.won@mfu.ac.th
}

\begin{abstract}
Men who have sex with men (MSM) are at higher risk for co-infection with hepatitis B virus (HBV), hepatitis $\mathrm{C}$ virus (HCV) than the general population. The co-infection accelerates disease progression reciprocally. This study purposed to investigate the prevalence and the associated factors with HBV and/or HCV co-infection with HIV among MSM in Northern region Thailand in 2015. A case-control study design was conducted by a hospital-based in anti-retroviral (ARV) clinic. 31 of 51 hospitals in the Phayao, Chiang Mai and Chiang Rai province were recruited in this study. The result was found $\mathbf{3 7 4}$ total participants who were registered patients in ARV clinic inPhayao84 cases (22.5\%), Chiang Mai 170 (45.5\%) and Chiang Rai120 (32.1\%). The participants were 30-43 year old (47.1\%), followed by $16-29$ years old $(29.9 \%)$, and $44-57$ years old $(19.8 \%)$. The majority of occupational were employee $(50.0 \%)$, follow by merchant $(24.9 \%)$ and agriculturist $(9.4 \%)$. The univariate analysis with a significant $\alpha$ level of 0.05 were found six factors had significantly association with HBV and/or HCV with HIV co-infection including (1) age range (2) smoking behavior (3) tongue piercing, (4) Stavudine drug taking, (5) Tenofivir drug taking and (6) Nevirapine drug taking. Moreover, the binary logistic regression at a significant $\alpha$ level of $\mathbf{0 . 0 5}$, after controlling for all possible confounding factors, were found four factors significantly association with co-infection including (1) age between 30-43 year old had the risk of co-infection 2.42 times [odds ratio $(\mathrm{OR})=2.42,95 \%$ confidence interval $(\mathrm{Cl})=1.14-5.14$ ] higher than those who had age range 16-29 year old, (2) smoking behavior in the participants had the risk of co-infection 2.05 times (OR $=2.05,95 \% \mathrm{Cl}=1.12-3.76$ ) higher than no smoking behavior. (3)Tongue piercing in the participants had the risk of co-infection 6.82 times $(\mathrm{OR}=6.70,95 \% \mathrm{Cl}=1.12-40.19)$ higher than no tongue piercing and (4) Tenofivir drug taking had the risk of co-infection $4.30(\mathrm{OR}=4.30,95 \% \mathrm{Cl}=2.37-7.84)$ more than no taking drug. Therefore, enhancing the surveillance and targeted prevention of viral hepatitis including improved disease prevention, vaccination coverage, sanitation and food safety, safer sex and efficiency healthcare delivery,
\end{abstract}

Keywords: The human immunodeficiency virus (HIV), Hepatitis B Virus (HBV), Hepatitis C Virus (HCV), Coinfection, Men who have sex with men (SMS).

\section{INTRODUCTION}

HIV/AIDS remains one of the world's most significant public health challenges. In 2015, World Health
Organization estimated approximately 36.7 million people living with HIV, 2.1 million people were the newly infected 
and 1.1 million people were died caused of AIDS(World Health Organization, 2016). HIV-positive persons who become infected with HBV or HCV are at increased risk for developing chronic hepatitis. In addition, persons who are co-infected with HIV and hepatitis can have serious medical complications, including an increased risk for liver-related morbidity and mortality (Centers for Disease Control and Prevention, 2016).

HIV and Hepatitis B virus (HBV) are bloodborne viruses transmitted primarily through sexual intercourse and injection drug use. Hepatitis $\mathrm{C}$ virus (HCV) is a bloodborne virus transmitted through direct contact with the blood of an infected person (Centers for Disease Control and Prevention, 2016).WHO estimated that HCV affects $2-15 \%$ of people living with HIV worldwide and that chronic HBV infection affects an estimated $5-20 \%$ of people living with HIV. The global estimate of burden of HIV-HCV co-infection and HBV-HCV co-infection are $2.75,2.6$ million respectively. The burden of these coinfections are greatest in the African and South East Asia Regions (World Health Organization, 2017).

In Thailand, since 1984 to September 2012, there had been 276,947 cumulative numbers of cases with sexual intercourse being the major route of transmission. In 2004, Sungkanuparph et al., determined the prevalence and risk factors of $\mathrm{HBV}$ and $\mathrm{HCV}$ co-infection in HIVinfected patients who were cared in March 2003 at Ramathibodi Hospital.

The prevalence of $\mathrm{HBV}$ and $\mathrm{HCV}$ co-infection in $\mathrm{HIV}$ was found $8.7 \%$ and $7.8 \%$ respectively. History of intravenous drug use was associated with both HBV and HCV co-infection ( $p<0.001)$. HCV co-infection group was also associated with male gender $(p=0.002)$ and elevated serum alanine transaminase $(A L T)$ level $(p=$ 0.0003). The co-infections are rapidly increased especially men who have sex with men (MSM) and people who inject drugs (PWID). (Sungkanuparph et al., 2004)

Men who have sex with men MSM) are at higher risk for co-infection with hepatitis B virus (HBV), hepatitis C virus (HCV) than the general population. The co-infection accelerates disease progression reciprocally. This study purposed to investigate the prevalence and the associated factors with HBV and/or HCV co-infection with HIV among MSM in Northern region Thailand in 2015 (Klaus et al, 2015).Chiang Rai Province in Northern Thailand has the highest HIV/AIDS prevalence of HIV infection.

The total number of HIV/AIDS patients from 1988 to October 2012 reported from Chiang Rai Provincial Public Health Office was 34,352 with 15,402 deaths. Information regarding prevalence and risk factors of $\mathrm{HBV}$ and $\mathrm{HCV}$ co-infection with HIV in Thailand is limited especially in MSM group (Apidechkul et al, 2016)

Therefore, this study purposed to investigate the influencing factors with $\mathrm{HBV}$ and $\mathrm{HCV}$ co-infection with HIV among MSM in Northern region Thailand.

\section{MATERIALS AND METHODS}

\section{Study design}

A case-control study design was conducted by a hospitalbased in MSM who have diagnosed with HIV infection until 2015. Cases were patients who HBV and/or HCV co-infection with HIV infected. HBV and HCV infection were confirmed by having one or more positive tests for $\mathrm{HBV}$ and HCV serology. Controls were those with all negative tests for HBV and HCV.

\section{Study site and study samples}

31 of 51 hospitals in the Chiang Mai, Chiang Rai and Phayao province where were purposive selected and willing to participate in this study. In Phayao province were 6 districts including Chiang Come, Pong, Chun, Chiang muan, Dok Khamtai, and Mae Jai. In Chiang Mai province were 14districts including San Kamphaeng, San Sai, Saraphi, Chom Thong, San Pa Tong, Doi Tao, Doi Saket, Hang Dong, Fang, Phrao, Mae Taeng, Hot, Omkoi and DoiLo. In Chiang Rai province were 11districts including Mae Chan, Mae Sai, Weing Chiang Rung, Phan, Mae Suai, Wiang Pa Pao, Khun Tan, Chiang Khong, Weing Chai, Mae Laos, Phaya Meng Rai.

The samples of this study were MSM patients who were first diagnosed with HIV positive before and until 2015 and lived in Phayao, Chiang Mai and Chiang Rai province at least 2 years before the commencement of the study, and had visited at least once in the selected antiretroviral (ARV)clinics.

\section{Sample size estimate}

The sample size was calculated by using an alpha at $5.0 \%$ and $80.0 \%$ for the power of the test. The calculation yielded a figure of 278 cases plus $10.0 \%$ to account for loss to follow up resulting in a final count of 80 cases and 237 controls. All participants knew they were MSM with HIV positive, and gave informed consent prior to participating in the study. Both cases and controls were selected from the lists of HIV patients from the target hospitals by purposive selecting technique. Only those who had met criteria were recruited into the study.

\section{Study instruments}

A structured questionnaire was interviewed the participants about socio-demographic characteristics, medical history, risk behaviors and sexual behaviors. Socio-demographics were age, gender, marital status, religion, area of residence, number of family members, education level, occupational and monthly income. 


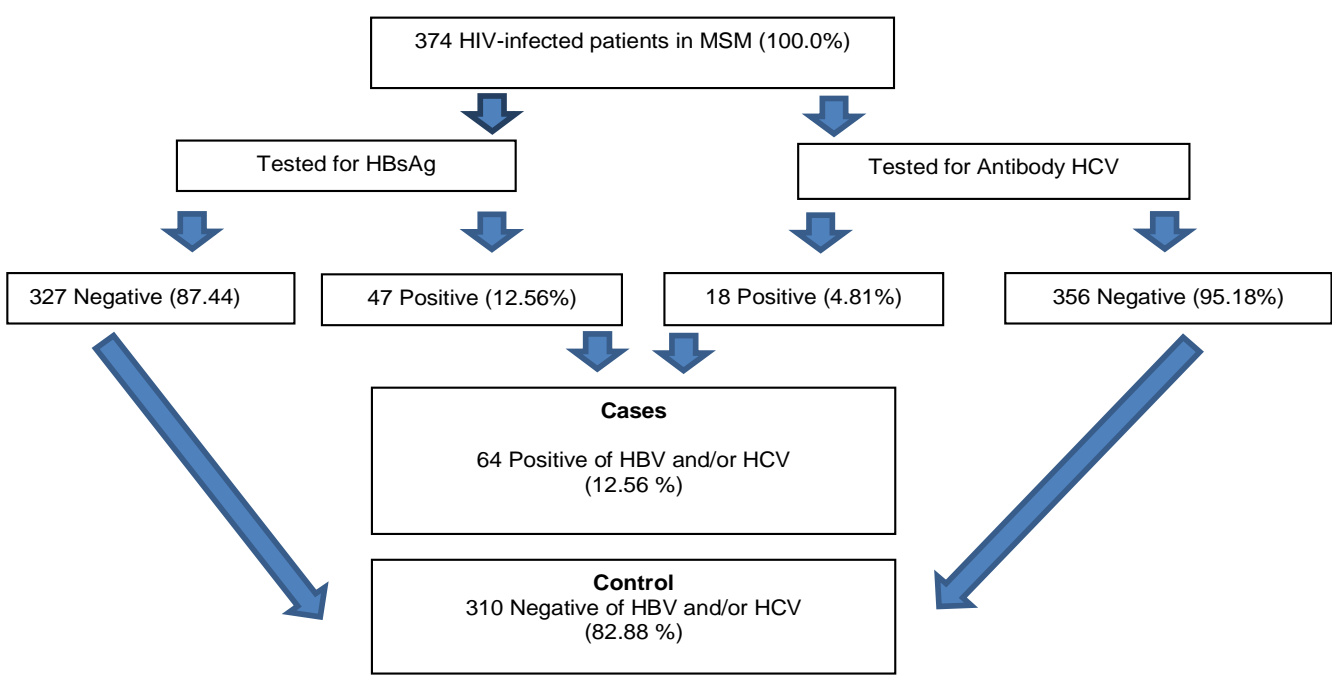

Figure 1.Selection of cases and controls

Medical history was interviewed about history of blood transfusion, hemodialysis, jaundice, HBV and HCV vaccination, use of ARV drugs, recent CD4 cell count, length of HIV infection and presence of any co morbidity. Co-infection risk behavior were interviewed about history of intra drug use, history of drug abuse throwing inhalation or orally, tattooing, piercing, alcohol use, and smoking. The participants were also interviewed about ages at first sexual intercourse, sexual orientation, history of sexual transmitted diseases, history of commercial sex work, number of sexual partners, and history of oral and anal sex of the use of condoms. The validity of questionnaire was validated by three external experts before used. It was adjusted if the score was less than 0.5 in the Item Objective Congruence Index method. The reliability of the questionnaire was tested among 30 patients with test-retest reliability method before use in the field with a Cronbach's alpha of 0.81 .

\section{Laboratory methods}

Five milliliters of venous blood was obtained from each subject and the hepatitis B surface antigen $(\mathrm{HBsAg})$ and hepatitis $\mathrm{C}$ antibody (Anti-HCV) were examined (Figure 1). $\mathrm{HBS} \mathrm{Ag}$ and $\mathrm{HCV} \mathrm{Ab}+$ were detected by NIBSC 00/588; WHO 2nd International Standard for HBsAg, subtype adw2, genotype $\mathrm{A}$; UI/mL with $99.9 \%$ specificity and $99.8 \%$ sensitivity (Muhlbacher et al., 2008) and hepatitis $\mathrm{C}$ antibody (Anti-HCV) by cobas e 411 analyzer, Elecsys $\AA 2010$ analyzer: 4.2 - $5.2 \%$ with $100.0 \%$ sensitivity and $99.6 \%$ hospitalized patients clinical specificity (Esteban, 2013 )

\section{Statistical analysis}

Descriptive statistics (frequencies, percentages, means, and standard deviations) were used to describe the general characteristics of the participants. Univariate analysis was used to identify factors associated of HBV and /or HCV with HIV co-infection at $\alpha=0.05$. All significant variables were included in model for Binary logistic regression analysis. Binary logistic regression analysis was conducted to identify the associated factors of HBV and /or HCV with HIV co-infection by controlling all possible confounding factors.

\section{Ethical considerations}

This study was approved by the Ethics in Human Research Committee of Mae Fah Luang University (No. $5 / 2557$ ). Permission of performing the study was also obtained from the Provincial Chief of Public Health Office for Phayao, Chiang Mai and Chiang Rai.

\section{RESULTS}

Total participants were 374 HIV registered patients among MSM group from 31 hospitals in Phayao, Chiang Mai, Chiang Rai province, Thailand, 2015 who were recruited based on the inclusion criteria. Of these cases were registered ARV clinic patients in Phayao84 cases (22.45\%), Chiang Mai 170 cases (45.45\%) and Chiang Rai120 cases (32.08\%). The general characteristic of all participants were $30-43$ year old $(47.1 \%), 16-29$ years old $(29.9 \%)$, and $44-57$ years old $(19.8 \%)$. The majority of occupational were employee $(50.0 \%)$, merchant $(24.9 \%)$ and agriculturist $(9.4 \%)$. Moreover, they were graduated secondary school $(40.4 \%)$, low income per month $(89.0 \%)$, Thai race $(98.9 \%)$, Buddhism $(97.6 \%)$, single $(91.7 \%), 10-18$ years old of the $1^{\text {st }}$ sexual intercourse $(85.6 \%)$, equal and more than 1 persons of the partner $(50.3 \%)$, not used condom $(90.6 \%)$, CD4 level more than 250 (70.3), 1-10 duration years of HIV infection (78.5\%), 1-4 duration years of receiving ARV drug. (Table1) 
034 J. Med. Med. Sci.

Table 1: The general characteristics of HIV/AIDS patients in MSM group in the Northern region of Thailand, 2015

\begin{tabular}{|c|c|c|c|c|c|}
\hline \multirow{3}{*}{$\begin{array}{l}\text { Characteristics } \\
\text { All Subjects (MSM) }\end{array}$} & \multirow{3}{*}{$\begin{array}{l}\text { Totaln (\%) } \\
374(100) \\
\end{array}$} & \multicolumn{3}{|c|}{ Provinces } & \multirow{3}{*}{$\begin{array}{c}\text { P-value } \\
0.00^{\star}\end{array}$} \\
\hline & & Phayaon (\%) & Chiang Main (\%) & Chiang Rain (\%) & \\
\hline & & $84(22.45)$ & $170(45.45)$ & $120(32.08)$ & \\
\hline \multicolumn{6}{|l|}{ Age } \\
\hline$-16-29$ & $112(29.9)$ & $25(29.8)$ & $62(36.5)$ & $25(20.8)$ & 0.13 \\
\hline$-30-43$ & $176(47.1)$ & 36 (42.9) & 74 (43.5) & $66(55.0)$ & \\
\hline$-44-57$ & $74(19.8)$ & 19 (22.6) & $30(17.6)$ & $25(20.8)$ & \\
\hline$-58-71$ & $12(3.2)$ & $4(4.8)$ & $4(2.4)$ & $4(3.3)$ & \\
\hline \multicolumn{6}{|l|}{ Occupational } \\
\hline - Civil Servant & 12 (3.2) & $3(3.6)$ & $4(2.4)$ & $5(4.2)$ & 0.00 \\
\hline - Agriculturist & $35(9.4)$ & 17 (20.2) & $3(1.8)$ & $15(12.5)$ & \\
\hline - Employee & $187(50.0)$ & $40(47.6)$ & $86(50.6)$ & $61(50.8)$ & \\
\hline - Merchant & $93(24.9)$ & $18(21.4)$ & 49 (28.8) & 26 (21.7) & \\
\hline - Unemployed & $32(8.6)$ & $5(6.0)$ & 22 (12.9) & $5(4.2)$ & \\
\hline - Student & $11(2.9)$ & $1(1.2)$ & $4(2.4)$ & $6(5.0)$ & \\
\hline - Monk & $4(1.1)$ & $0(0.0)$ & $2(1.2)$ & $2(1.7)$ & \\
\hline \multicolumn{6}{|l|}{ Education } \\
\hline - Un education & $8(2.1)$ & $2(2.4)$ & $4(2.4)$ & $2(1.7)$ & 0.65 \\
\hline - Primary School & $105(28.1)$ & $28(33.3)$ & $38(22.4)$ & 39 (32.5) & \\
\hline - Secondary School & $151(40.4)$ & $34(40.5)$ & $72(42.4)$ & $45(37.5)$ & \\
\hline - High Certification & $35(9.4)$ & $6(7.1)$ & 18 (10.6) & $11(9.2)$ & \\
\hline - Bachelor and Higher & $75(20.1)$ & $14(16.7)$ & $38(22.4)$ & $23(19.2)$ & \\
\hline \multicolumn{6}{|l|}{ Income } \\
\hline - 500-25,500 & $333(89.0)$ & 78 (92.9) & $149(87.6)$ & $106(88.3)$ & 0.18 \\
\hline$-25,501-50,500$ & $35(9.4)$ & $4(4.8)$ & $20(11.8)$ & $11(9.2)$ & \\
\hline$-50,501-75,500$ & $1(0.3)$ & $1(1.2)$ & $0(0.0)$ & $0(0.0)^{\prime}$ & \\
\hline$-75,501-100,500$ & $5(1.3)$ & $1(1.2)$ & $1(0.6)$ & $3(2.5)$ & \\
\hline \multicolumn{6}{|l|}{ Race } \\
\hline - Thai & $370(98.9)$ & $84(100.0)$ & 167 (98.2) & 119 (99.2) & 1.0 \\
\hline - Akha & $3(0.8)$ & $0(0.0)$ & $2(1.2)$ & $1(0.8)$ & \\
\hline - Karen & $1(0.3)$ & $0(0.0)$ & $1(0.6)$ & $0(0.0)$ & \\
\hline \multicolumn{6}{|l|}{ Religion } \\
\hline - Buddhism & $365(97.6)$ & $81(96.4)$ & $165(97.1)$ & 119 (99.2) & $0.38 \mathrm{a}$ \\
\hline - Christianity & $9(2.4)$ & $3(3.6)$ & $5(2.9)$ & $1(0.8)$ & \\
\hline \multicolumn{6}{|l|}{ Family Status } \\
\hline - Single & $343(91.7)$ & 78 (92.9) & $155(91.2)$ & $110(91.7)$ & $0.02 \mathrm{a}$ \\
\hline - Couple & $25(6.7)$ & $2(2.4)$ & $15(8.8)$ & $8(6.7)$ & \\
\hline - Widow & $2(0.5)$ & $2(2.4)$ & $0(0.0)$ & $0(0.0)$ & \\
\hline - Divorce & $1(0.3)$ & $1(1.2)$ & $0(0.0)$ & $0(0.0)$ & \\
\hline - Separated & $3(0.8)$ & $1(1.2)$ & $0(0.0)$ & $2(1.7)$ & \\
\hline \multicolumn{6}{|l|}{$\begin{array}{l}\text { Age of } 1^{\text {st }} \text { sexual } \\
\text { intercourse }\end{array}$} \\
\hline$-10-18$ & $320(85.6)$ & $74(88.1)$ & $144(84.7)$ & $102(85.0)$ & $0.58 \mathrm{a}$ \\
\hline$-19-27$ & $45(12.0)$ & $10(11.9)$ & $20(11.8)$ & $15(12.5)$ & \\
\hline$-28-36$ & $9(2.4)$ & $0(0.0)$ & $6(3.5)$ & $3(2.5)$ & \\
\hline \multicolumn{6}{|l|}{ Number of partner } \\
\hline - No partner & $186(49.7)$ & $44(52.4)$ & $81(47.6)$ & $61(50.8)$ & 0.84 \\
\hline - 1 person & $120(32.1)$ & $28(33.3)$ & $55(32.4)$ & $37(30.8)$ & \\
\hline - >= 2 persons & $68(18.2)$ & $12(14.3)$ & $34(0.0)$ & $22(18.3)$ & \\
\hline \multicolumn{6}{|l|}{ Condom use behavior } \\
\hline - Not used & $339(90.6)$ & $73(86.9)$ & $153(90.0)$ & $113(94.2)$ & 0.20 \\
\hline - Always used & $35(9.4)$ & $11(13.1)$ & $17(10.0)$ & $7(5.8)$ & \\
\hline \multicolumn{6}{|l|}{ Addictive substance } \\
\hline - Not used & $336(89.8)$ & $71(84.5)$ & $149(87.6)$ & $116(96.7)$ & $0.01^{*}$ \\
\hline - Used & $38(10.2)$ & $13(15.5)$ & $21(12.4)$ & $4(3.3)$ & \\
\hline \multicolumn{6}{|l|}{ Smoking Behavior } \\
\hline - No smoking & $191(51.1)$ & $44(52.4)$ & $86(50.6)$ & $61(50.8)$ & 0.96 \\
\hline - Smoking & $183(48.9)$ & $40(47.6)$ & $84(49.4)$ & 59 (49.2) & \\
\hline
\end{tabular}


Table 1 continue

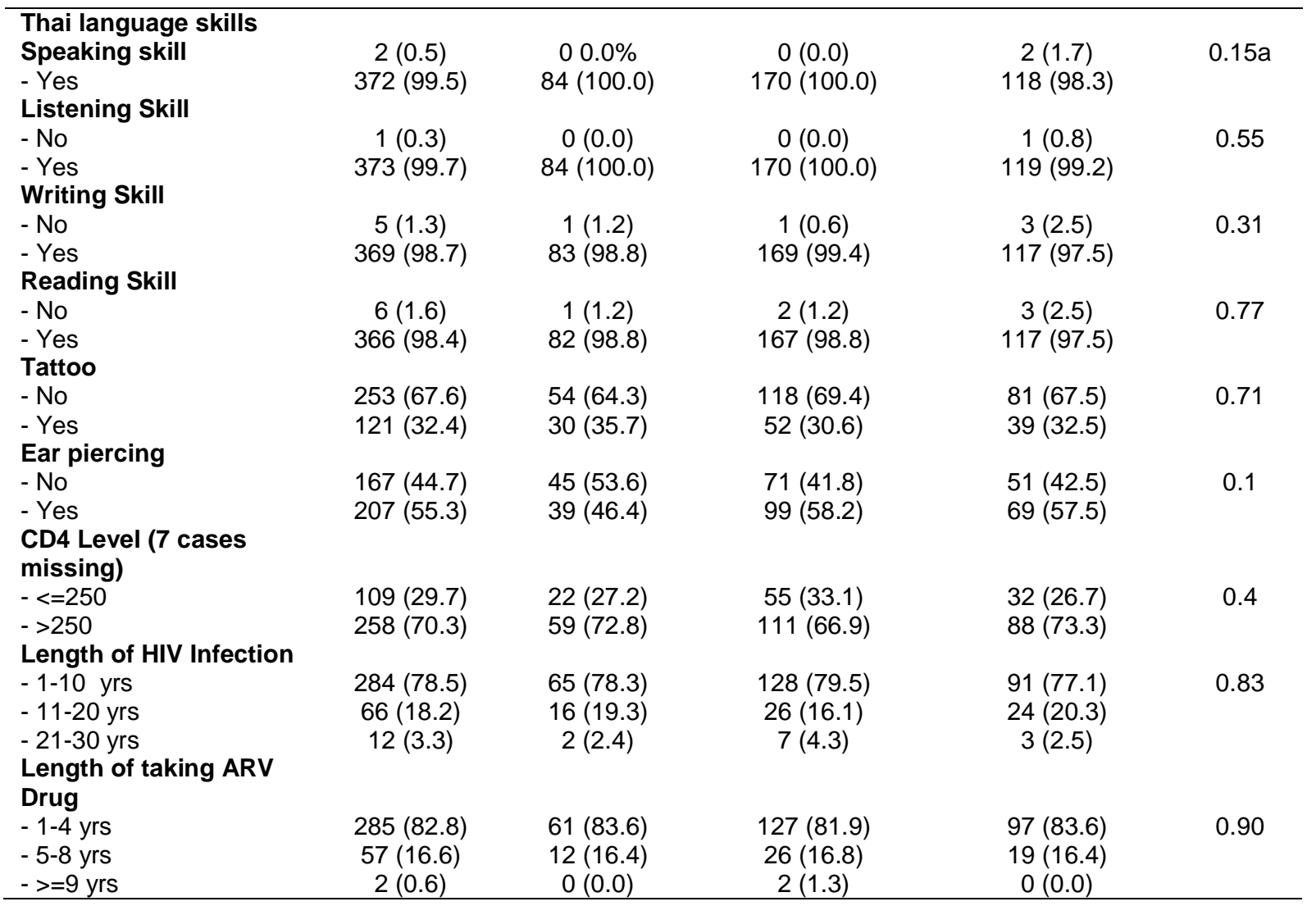

The prevalence of $\mathrm{HBsAg}$ positive and $\mathrm{HCV} \mathrm{Ab}+$ positive with HIV infected among MSM were $12.6 \%$ (47/374) and 4.8\% (18/374). Also, the prevalence of HBsAg positive and/or HCV Ab+ positive with HIV infected was $17.1 \%(64 / 374)$ who were assigned as the cases group, while $82.9 \%$ (310/374) who were negative both HBsAg and $\mathrm{HCV} \mathrm{Ab+} \mathrm{as} \mathrm{the} \mathrm{control} \mathrm{group.} \mathrm{The}$ important finding of risk behaviors in the cases group were always used condom (87.5\%), used addictive substance $(10.9 \%)$, smoking $(62.5 \%)$, drinking $(51.6 \%)$, tattoo $(34.4 \%)$, eye piercing $(57.8 \%)$, CD4 level equal and less than 250 (33.9\%), 1-10 years duration of HIV infection (84.7\%), 1-4 years duration of ARV drug $(89.7 \%)$. Finally, this study was also found high level of knowledge $(70.3 \%)$, high level of attitude (76.6\%) and moderate level of practice (56.2\%) in term of HIV, HBV and HCV co-infection of the participants (Table 2).

The univariate analysis (simple logistic regression) with a significant level of $\alpha$ at 0.05 were found six factors had significantly association with $\mathrm{HBV} / \mathrm{HCV} / \mathrm{HIV}$ coinfection. There were (1) age range, (2) smoking behavior (3) tongue piercing, (4) Stavudine receiving, (5) Tenofivir receiving and (6) Nevirapine receiving. In the participants who had age range between 30-43 year old had the risk of co-infection 1.99 times [odds ratio $(\mathrm{OR})=1.99$, 95\% confidence interval $(C l)=1.03-3.87 .14]$ higher than those who had age range 16-29 year old. Smoking behavior in the participants had the risk of co-infection 1.95 times (OR $=1.95,95 \% \mathrm{Cl}=1.12-3.38)$ higher than no smoking behavior. Tongue piercing in the participants had the risk of co-infection 6.82 times (OR $=6.82,95 \% \mathrm{Cl}=1.49$ 31.26) higher than no tongue piercing. The interesting finding which significantly associated with the co-infection in this study were antiviral HIV drug receiving which including Stavudine, Tenofivir and Nevirapine. No receiving Stavudine and Nevirapine in the participants had the risk of co-infection 2.71 times (OR $=2.71,95 \%$ $\mathrm{Cl}=1.33-5.55)$ and 2.08 times $(\mathrm{OR}=2.08,95 \% \mathrm{Cl}=$ 1.21-3.59) higher than the participants who received Stavudine and Nevirapine respectively. On the other hand, the participants who received Tenofivir had the risk of co-infection 4.09 (OR $=2.71,95 \% \mathrm{Cl}=1.33-5.55)$ more than no received this drug (Table 3 ).

Binary logistic regression at a significant a level of0.05, after controlling for all possible confounding factors, four factors were found significantly association with co-infection. The firstly, the participants who had age range between 30-43 year old had the risk of coinfection 2.42 times [odds ratio $(\mathrm{OR})=2.42$, 95\% confidence interval $(\mathrm{Cl})=1.14-5.14]$ higher than those who had age range 16-29 year old. Secondly, smoking behavior in the participants had the risk of co-infection 
036 J. Med. Med. Sci.

Table 2: The general characteristics of the MSM HIV/AIDS patients

\begin{tabular}{|c|c|c|c|c|}
\hline Characteristics & Totaln (\%) & HBsAg+n (\%) & HCV Ab+n (\%) & $\begin{array}{c}\text { Cases } \\
\text { (HBsAg+ and/orHCVAb+)n (\%) }\end{array}$ \\
\hline All Subjects & $374(100)$ & $47(12.57 \%)$ & $18(4.81 \%)$ & $64(17.11 \%)$ \\
\hline \multicolumn{5}{|l|}{ Province } \\
\hline - Phayao & $84(22.5)$ & $12(25.5)$ & $3(16.7)$ & $14(21.9)$ \\
\hline - Chiang Mai & $170(45.5)$ & 15 (31.9) & $11(61.1)$ & $26(40.6)$ \\
\hline - Chiang Rai & $120(32.1)$ & $20(42.6)$ & $4(22.2)$ & $24(37.5)$ \\
\hline \multicolumn{5}{|l|}{ Age } \\
\hline$-16-29$ & $112(29.9)$ & $10(21.3)$ & $4(22.2)$ & $14(21.9)$ \\
\hline$-30-43$ & $176(47.1)$ & $29(61.7)$ & $11(61.1)$ & 39 (60.9) \\
\hline$-44-57$ & $74(19.8)$ & $6(12.8)$ & $3(16.7)$ & $9(14.1)$ \\
\hline - 58-71 - - l & $12(3.2)$ & $2(4.3)$ & $0(0.0)$ & $2(3.1)$ \\
\hline \multicolumn{5}{|l|}{ Occupational } \\
\hline - Civil Servant & $12(3.2)$ & $0(0.0)$ & $0(0.0)$ & $0(0.0)$ \\
\hline - Agriculturist & $35(9.4)$ & $5(10.6)$ & $1(5.6)$ & $6(9.4)$ \\
\hline - Employee & $187(50.0)$ & $19(40.4)$ & $10(55.6)$ & $29(45.3)$ \\
\hline - Merchant & $93(24.9)$ & $16(34.0)$ & $4(22.2)$ & $20(31.2)$ \\
\hline - Unemployed & $32(8.6)$ & $6(12.8)$ & $3(6.7)$ & $8(12.5)$ \\
\hline - Student & $11(2.9)$ & $1(2.1)$ & $0(0.0)$ & $1(1.6)$ \\
\hline - Monk & $4(1.1)$ & $0(0.0)$ & $0(0.0)$ & $0(0.0)$ \\
\hline \multicolumn{5}{|l|}{ Education } \\
\hline - Un education & $8(2.1)$ & $1(2.1)$ & $0(0.0)$ & $1(1.6)$ \\
\hline - Primary School & $105(28.1)$ & $13(27.7)$ & $4(22.2)$ & $17(26.6)$ \\
\hline - Secondary School & $151(40.4)$ & 18 (38.3) & $10(55.6)$ & $28(43.8)$ \\
\hline - High Certification & $35(9.4)$ & $5(10.6)$ & $1(5.6)$ & $6(9.4)$ \\
\hline - Bachelor and Higher & $75(20.1)$ & $10(21.3)$ & 3 (16.7) & $12(18.8)$ \\
\hline \multicolumn{5}{|l|}{ Income } \\
\hline - 500-25,500 & $333(89.0)$ & $43(91.5)$ & $18(100.0)$ & $60(93.8)$ \\
\hline - 25,501-50,500 & $35(9.4)$ & $3(6.4)$ & $0(0.0)$ & $3(4.7)$ \\
\hline - 50,501 - 75,500 & $1(0.3)$ & $1(2.1)$ & $0(0.0)$ & $1(0.6)$ \\
\hline - 75,501- 100,500 & $5(1.3)$ & $0(0.0)$ & $0(0.0)$ & $0(0.0)$ \\
\hline \multicolumn{5}{|l|}{ Race } \\
\hline - Akha & $3(0.8)$ & $0(0.0)$ & $0(0.0)$ & $0(0.0)$ \\
\hline - Karen & $1(0.3)$ & $0(0.0)$ & $0(0.0)$ & $0(0.0)$ \\
\hline - Thai & $370(98.9)$ & $47(100.0)$ & $18(100.0)$ & $64(100.0)$ \\
\hline \multicolumn{5}{|l|}{ Religion } \\
\hline - Buddhism & $365(97.6)$ & $47(100.0)$ & $18(100.0)$ & $64(100.0)$ \\
\hline - Christianity & $9(2.4)$ & $0(0.0)$ & $0(0.0)$ & $0(0.0)$ \\
\hline \multicolumn{5}{|l|}{ Family Status } \\
\hline - Single & $343(91.7)$ & $43(91.5)$ & $17(94.4)$ & 59 (92.2) \\
\hline - Couple & $25(6.7)$ & $3(6.4)$ & $0(0.0)$ & $3(4.7)$ \\
\hline - Widow & $2(0.5)$ & $0(0.0)$ & $0(0.0)$ & $0(0.0)$ \\
\hline - Divorce & $1(0.3)$ & $0(0.0)$ & $0(0.0)$ & $0(0.0)$ \\
\hline - Separated & $3(0.8)$ & $1(2.1)$ & $1(5.6)$ & $2(3.1)$ \\
\hline \multicolumn{5}{|l|}{$\begin{array}{l}\text { Age of } 1^{\text {st }} \text { sexual } \\
\text { intercourse }\end{array}$} \\
\hline$-10-18$ & $253(67.6)$ & $31(66.0)$ & $13(72.2)$ & $44(68.8)$ \\
\hline - 19-27 & $111(29.7)$ & $15(31.9)$ & $5(27.8)$ & $19(29.7)$ \\
\hline$-28-36$ & $10(2.7)$ & $1(2.1)$ & $0(0.0)$ & $1(1.6)$ \\
\hline \multicolumn{5}{|l|}{ Number of partner } \\
\hline - No have partner & $186(49.7)$ & $28(59.6)$ & $9(50.0)$ & $36(56.2)$ \\
\hline - 1 person & $120(32.1)$ & $13(27.7)$ & $5(27.8)$ & $18(28.1)$ \\
\hline$->=2$ persons & $68(18.2)$ & $6(12.8)$ & $4(22.2)$ & $10(15.6)$ \\
\hline \multicolumn{5}{|l|}{ Condom use behavior } \\
\hline - Not used & $35(9.4)$ & $3(6.4)$ & $5(27.8)$ & $8(12.5)$ \\
\hline - Always used & $339(90.6)$ & $44(93.6)$ & $13(72.2)$ & $56(87.5)$ \\
\hline \multicolumn{5}{|l|}{ Addictive substance } \\
\hline - Not used & $336(89.8)$ & $43(91.5)$ & 15 (83.3) & $57(89.1)$ \\
\hline - Used & $38(10.2)$ & $4(8.5)$ & $3(16.7)$ & $7(10.9)$ \\
\hline
\end{tabular}


Table 2 continue

\begin{tabular}{|c|c|c|c|c|}
\hline \multicolumn{5}{|c|}{ Smoking Behavior } \\
\hline - No smoking & $191(51.1)$ & $21(44.7)$ & $3(16.7)$ & $24(37.5)$ \\
\hline - Smoking & $183(48.9)$ & 26 (55.3) & 15 (83.3) & $40(62.5)$ \\
\hline \multicolumn{5}{|c|}{ Drinking Behavior } \\
\hline - No drinking & $150(40.1)$ & $21(44.7)$ & $11(61.1)$ & $31(48.4)$ \\
\hline - Drinking & $224(59.9)$ & $26(55.3)$ & 7 (38.9) & $33(51.6)$ \\
\hline \multicolumn{5}{|l|}{ Tattoo } \\
\hline - No & $253(67.6)$ & $32(68.1)$ & $10(55.6)$ & $42(65.6)$ \\
\hline - Yes & $121(32.4)$ & $15(31.9)$ & $8(44.4)$ & $22(34.4)$ \\
\hline \multicolumn{5}{|l|}{ Ear piercing } \\
\hline - No & $167(44.7)$ & $23(48.9)$ & $4(22.2)$ & $27(42.2)$ \\
\hline - Yes & $20(55.3)$ & $24(51.1)$ & $14(77.8)$ & $37(57.8)$ \\
\hline \multicolumn{5}{|c|}{$\begin{array}{l}\text { CD4 Level ( } 7 \text { cases } \\
\text { missing) }\end{array}$} \\
\hline$-<=250$ & $109(29.7)$ & $14(31.1)$ & $8(44.4)$ & $21(33.9)$ \\
\hline$->250$ & $258(70.3)$ & 31 (68.9) & $10(55.6)$ & $41(66.1)$ \\
\hline \multicolumn{5}{|l|}{ Length of HIV } \\
\hline \multicolumn{5}{|c|}{$\begin{array}{l}\text { Infection } \\
\text { (12 case missing) }\end{array}$} \\
\hline$-1-10$ yrs & $284(78.5)$ & 37 (82.2) & $14(93.3)$ & $50(84.7)$ \\
\hline$-11-20 \mathrm{yrs}$ & $66(18.2)$ & $8(17.8)$ & $1(6.7)$ & $9(15.3)$ \\
\hline$-21-30$ yrs & $12(3.3)$ & $0(0.0)$ & $0(0.0)$ & $0(0.0)$ \\
\hline \multicolumn{5}{|c|}{ Length of taking ARV } \\
\hline \multicolumn{5}{|l|}{ Drug (30 case } \\
\hline$-1-4$ yrs & $285(82.8)$ & $39(88.6)$ & $14(93.3)$ & $52(89.7)$ \\
\hline$-5-8$ yrs & $57(16.6)$ & $5(11.4)$ & $1(6.7)$ & $6(10.3)$ \\
\hline$->=9$ yrs & $2(0.6)$ & $0(0.0)$ & $0(0.0)$ & $0(0.0)$ \\
\hline \multicolumn{5}{|l|}{ Knowledge Level } \\
\hline - Low level & $8(2.1)$ & $0(0.0)$ & $1(5.6)$ & $1(1.6)$ \\
\hline - Moderate level & $89(23.8)$ & $14(29.8)$ & $4(22.2)$ & $18(28.1)$ \\
\hline - High Level & $277(74.1)$ & $33(70.2)$ & $13(72.2)$ & $45(70.3)$ \\
\hline \multicolumn{5}{|l|}{ Attitude Level } \\
\hline - Low level & $14(3.7)$ & $1(2.1)$ & $1(5.6)$ & $2(3.1)$ \\
\hline - Moderate level & $107(28.6)$ & $8(17.0)$ & $5(27.8)$ & $13(20.3)$ \\
\hline - High Level & $253(67.6)$ & 38 (80.9) & $12(66.7)$ & $49(76.6)$ \\
\hline \multicolumn{5}{|l|}{ Practice Level } \\
\hline - Low level & $9(2.4)$ & $0(0.0)$ & $1(5.6)$ & $1(1.6)$ \\
\hline - Moderate level & $214(57.2)$ & $26(55.3)$ & $10(55.6)$ & $36(56.2)$ \\
\hline - High Level & $151(40.4)$ & $21(44.7)$ & 7 (38.9) & $27(42.2)$ \\
\hline
\end{tabular}

2.05 times $(\mathrm{OR}=2.05,95 \% \mathrm{Cl}=1.12-3.76)$ higher than no smoking behavior. Thirdly, tongue piercing in the participants had the risk of co-infection 6.82 times $(\mathrm{OR}=$ $6.70,95 \% \mathrm{Cl}=1.12-40.19)$ higher than no tongue piercing.

Finally, the participants who received Tenofivir had the risk of co-infection $4.30(\mathrm{OR}=4.30,95 \% \mathrm{Cl}=2.37-7.84)$ more than no received this drug (Table 4).

\section{DISCUSSION}

In this study, the total cases of HIV infection in MSM in the northern region were 374 participants who were registered ARV clinic and willing to participate in 31 hospitals in Phayao, Chiang Mai and Chiang Rai province, Thailand in 2015. The prevalence of HBV/HIV and HCV/HIV co-infection was $12.57 \%$ (47/374) and $4.81 \%(18 / 374)$ respectively.

Moreover, the prevalence rate of $\mathrm{HBV}$ and/or HCV with HIV co-infection was $17.11 \%$. HIV infection in MSM group refer to CDC fact sheet in 2014 of HIV among gay and bisexual men, Overall, MSM account was 56 percent (estimated 615,400 persons) of the estimated 1.1 million people living with HIV in the United States and more than 70 percent, or an estimated 26,200 infections of all new HIV infections each year.

Moreover, CDC estimated that four percent of men in the United States were MSM, the rate of new HIV diagnoses among them is more than 44 times that of other men (rate ranges from 522 to 989 per 100,000 MSM compared to 12 per 100,000 other men) (Centers for Disease Control and Prevention, 2017). These information of CDC fact sheet was similarly with the "Who 
038 J. Med. Med. Sci.

Table 3: Univariate analysis (Simple logistic regression) of factors associated of HBV and/or HVC co-infection with HIV of the MSM patients.

\begin{tabular}{|c|c|c|c|c|}
\hline \multirow{3}{*}{ Characteristics } & \multicolumn{2}{|c|}{ (Case) } & \multirow{3}{*}{ OR Crude(95\% CI) } & \multirow{3}{*}{ p-value } \\
\hline & \multicolumn{2}{|c|}{ HBV and/or HCV infection } & & \\
\hline & Non (\%) & Yes (\%) & & \\
\hline All Subjects = $374(100)$ & $310(82.9)$ & $64(17.1)$ & & \\
\hline \multicolumn{5}{|l|}{ Agerange } \\
\hline$-16-29$ & $98(87.5)$ & $14(12.5)$ & 1.00 & \\
\hline$-30-43$ & $137(77.8)$ & $39(22.2)$ & 1.99 (1.03-3.87) & $0.04^{*}$ \\
\hline$-44-57$ & $65(87.8)$ & $9(12.2)$ & $0.97(0.39-2.37)$ & 0.95 \\
\hline$-58-71$ & $10(83.3)$ & $2(16.7)$ & $1.40(0.28-7.06)$ & 0.68 \\
\hline \multicolumn{5}{|l|}{ Education } \\
\hline - Non education & $7(87.5)$ & $1(12.5)$ & 1.00 & \\
\hline - Primary School & $88(83.8)$ & $17(16.2)$ & $1.35(0.16-11.71)$ & 0.78 \\
\hline - Secondary School & $123(81.5)$ & $28(18.5)$ & $1.59(0.19-13.48)$ & 0.67 \\
\hline - High Certificate & $29(82.9)$ & $6(17.1)$ & $1.45(0.15-14.05)$ & 0.75 \\
\hline - Bachelor & $63(84.0)$ & $12(16.0)$ & $1.33(0.15-11.85)$ & 0.80 \\
\hline \multicolumn{5}{|l|}{$\begin{array}{l}\text { Age at first sexual } \\
\text { intercourse }\end{array}$} \\
\hline$-10-18$ & $209(82.6)$ & $44(17.4)$ & $1.90(0.23-15.34)$ & 0.55 \\
\hline$-19-27$ & $92(82.9)$ & $19(17.1)$ & $1.86(0.22-15.55)$ & 0.57 \\
\hline$-28-36$ & $9(90.0)$ & $1(10.0)$ & 1.00 & \\
\hline \multicolumn{5}{|l|}{ Number of partner } \\
\hline - No have partner & $150(80.6)$ & $36(19.4)$ & $1.024(0.44-2.37)$ & 0.96 \\
\hline - 1 partner & $102(85.0)$ & $18(15.0)$ & $1.392(0.65-2.99)$ & 0.40 \\
\hline - >=2 persons & $58(85.3)$ & $10(14.7)$ & 1.00 & \\
\hline \multicolumn{5}{|l|}{ Condom use behavior } \\
\hline - Not used & $27(77.1)$ & $8(22.9)$ & $1.497(0.65-3.47)$ & 0.35 \\
\hline - Always used & $283(83.5)$ & $56(16.5)$ & 1.00 & \\
\hline \multicolumn{5}{|l|}{ Addictive substance } \\
\hline - Not used & $279(83.0)$ & $57(17.0)$ & $0.91(0.38-2.16)$ & 0.82 \\
\hline - Always used & $31(81.6)$ & $7(18.4)$ & 1.00 & \\
\hline \multicolumn{5}{|l|}{ Smoking Behavior } \\
\hline - No smoking & $167(87.4)$ & $24(12.6)$ & 1.00 & \\
\hline - Smoking & $143(78.1)$ & $40(21.9)$ & $1.95(1.12-3.38)$ & $0.02^{*}$ \\
\hline \multicolumn{5}{|l|}{ Tattoo } \\
\hline - No & $211(83.4)$ & $42(16.6)$ & 1.00 & \\
\hline - Yes & $99(81.8)$ & 22 (18.2) & $1.12(0.63-1.97)$ & 0.70 \\
\hline \multicolumn{5}{|l|}{ Ear piercing } \\
\hline - No & $140(83.8)$ & $27(16.2)$ & 1.00 & \\
\hline - Yes & $170(82.1)$ & 37 (17.9) & $1.13(0.65-1.95)$ & 0.66 \\
\hline \multicolumn{5}{|l|}{ Tongue piercing } \\
\hline - No & $307(83.7)$ & $60(16.3)$ & 1.00 & \\
\hline - Yes & $3(42.9)$ & $4(57.1)$ & $6.82(1.49-31.26)$ & $0.01^{*}$ \\
\hline \multicolumn{5}{|l|}{$\begin{array}{l}\text { Stavudine drug taking } \\
\text { ( } 5 \text { cases missing) }\end{array}$} \\
\hline - No & $203(79.0)$ & $54(21.0)$ & $2.71(1.33-5.55)$ & $0.006^{*}$ \\
\hline - Yes & $102(91.1)$ & $10(8.9)$ & 1.00 & \\
\hline \multicolumn{5}{|l|}{ Efavirenz drug taking } \\
\hline - No & $309(83.3)$ & $62(16.7)$ & 1.00 & \\
\hline - Yes & $1(33.3)$ & $2(66.7)$ & $9.97(0.89-111.64)$ & 0.06 \\
\hline \multicolumn{5}{|l|}{ Tenofivir drug taking } \\
\hline - No & $242(88.6)$ & $31(11.4)$ & 1.00 & \\
\hline - Yes & $63(65.6)$ & $33(34.4)$ & $4.09(2.33-7.18)$ & $0.001^{*}$ \\
\hline Nevirapine drug taking & & & & \\
\hline - No & $121(76.6)$ & $37(23.4)$ & 2.08 (1.21-3.59) & $0.008^{*}$ \\
\hline - Yes & $184(87.2)$ & $27(12.8)$ & 1.00 & \\
\hline $\begin{array}{l}\text { CD4 Level (Missing } 7 \\
\text { cases) }\end{array}$ & & & & \\
\hline$-<=250$ & $88(80.7)$ & $21(19.3)$ & $1.26(0.71-2.26)$ & 0.43 \\
\hline$->250$ & $217(84.1)$ & $41(15.9)$ & 1.00 & \\
\hline
\end{tabular}

* Significant $(\alpha<0.05)$ 
Table 3 continue

\begin{tabular}{|c|c|c|c|c|}
\hline \multicolumn{5}{|l|}{$\begin{array}{l}\text { Length of HIV Infection } \\
\text { (Missing } 12 \text { cases) }\end{array}$} \\
\hline$-1-8$ yrs & $234(82.4)$ & $50(17.6)$ & 1.00 & \\
\hline$-9-16$ yrs & $57(86.4)$ & $9(13.6)$ & $0.74(0.34-1.59)$ & 0.44 \\
\hline - 17-24 yrs & $12(100.0)$ & $0(0.0)$ & 0.00 & 0.99 \\
\hline \multicolumn{5}{|l|}{$\begin{array}{l}\text { Length of ARV Drug } \\
\text { taking }\end{array}$} \\
\hline$-0-15$ yrs & $233(81.8)$ & $52(18.2)$ & $1.971(0.81-4.83)$ & 0.14 \\
\hline$-16-30$ yrs & $53(89.8)$ & $6(10.2)$ & 1.00 & \\
\hline \multicolumn{5}{|l|}{ Knowledge Level } \\
\hline - Low level & 7 (87.5) & $1(12.5)$ & $1.36(0.16-11.31)$ & 0.78 \\
\hline - Moderate level & $71(79.8)$ & $18(20.2)$ & $1.78(0.21-15.36)$ & 0.60 \\
\hline - High Level & $232(83.8)$ & $45(16.2)$ & 1.00 & \\
\hline \multicolumn{5}{|l|}{ Attitude Level } \\
\hline - Low level & $12(85.7)$ & $2(14.3)$ & $1.441(0.31-6.65)$ & 0.64 \\
\hline - Moderate level & $94(87.9)$ & $13(12.1)$ & $0.83(0.17-4.13)$ & 0.82 \\
\hline $\begin{array}{l}\text { - High Level } \\
\text { Practice Level }\end{array}$ & $204(80.6)$ & $49(19.4)$ & 1.00 & \\
\hline - Low level & $8(88.9)$ & $1(11.1)$ & $0.93(0.54-1.61)$ & 0.79 \\
\hline - Moderate level & $178(83.2)$ & $36(16.8)$ & $0.57(.07-4.78)$ & 0.61 \\
\hline - High Level & $124(82.1)$ & $27(17.9)$ & 1.00 & \\
\hline
\end{tabular}

Table 4: Binary logistic regression of the associated factors of HBV and/or HVC coinfection with HIV of MSM patients

\begin{tabular}{lcccc}
\hline \multirow{2}{*}{ Characteristics } & \multicolumn{2}{c}{ HBV and/or HCV infection } & \multirow{2}{*}{$\begin{array}{c}\text { OR Crude } \\
\text { (95\% Cl) }\end{array}$} & p-value \\
\cline { 2 - 3 } All Subjects = 374 (100) & $\mathbf{N 1 0 ( 8 2 . 9 )}$ & $\mathbf{6 4}(\mathbf{1 7 . 1})$ & & \\
Age & $98(87.5)$ & $14(12.5)$ & 1.00 & \\
- 16-29 & $137(77.8)$ & $39(22.2)$ & $2.42(1.14-5.14)$ & $0.02^{*}$ \\
- 30-43 & $65(87.8)$ & $9(12.2)$ & $1.27(0.48-3.38)$ & 0.63 \\
- 44-57 & $10(83.3)$ & $2(16.7)$ & $1.42(0.26-7.96)$ & 0.69 \\
- 58-71 & & & & \\
Smoking Behavior & $167(87.4)$ & $24(12.6)$ & 1.00 & \\
- No smoking & $143(78.1)$ & $40(21.9)$ & $2.05(1.12-3.76)$ & $0.02^{*}$ \\
- Smoking & & & & \\
Tongue piercing & $307(83.7)$ & $60(16.3)$ & 1.00 & \\
- No & $3(42.9)$ & $4(57.1)$ & $6.70(1.12-40.19)$ & $0.04^{*}$ \\
- Yes & & & & \\
Tenofivir drug taking & $242(88.6)$ & $31(11.4)$ & 1.00 & \\
- No & $63(65.6)$ & $33(34.4)$ & $4.30(2.37-7.82)$ & $0.00^{*}$ \\
- Yes & & &
\end{tabular}

* Significant $(\alpha<0.05)$

are MSM in Thailand? How many? Do you know? " in article in Population and Development newsletter by Niphon Darawutmaprakorn who was the specialist of the institute for population and social research, Mahidon university. Base on network scale up, multiple source method and programmatic mapping technics were applied to estimate amount MSM in Thailand. He found around 3 percent of men (15-59 years old) in Thailand were MSM (Darawutmaprakorn, 2016). Therefore, MSM account in Thailand estimated 649,087 persons of 21,636, 240 persons. Moreover, this study was applied these information for estimating the prevalence rate of HIV infection in SMS group in area study. We estimated that the total MSM group in Phayao, Chiang Mai and Chiang Raiprovine were 8,631, 14,222 and 12,013, persons respectively (calculate by $3 \%$ of total mal population between 15-59 years old in each province) and the total MSM group in three provinces was 24,107 persons. Therefore, the prevalence rate of HIV infection in 2015 in study area was $3.17 \%(374 / 24,107)$ that was closely with the CDC that estimated $4 \%$ in USA, 2014, while the prevalence rate of HIV infection in 2015 in Phayao, Chiang Rai and Chiang Mai were 2.43\% (84/8,631), $1.42 \% \quad(170 / 12,013) \quad$ and $1.39 \% \quad(120 / 12,013)$ respectively.

$\mathrm{HIV} / \mathrm{HBV}$ co-infection in MSM. The prevalence rate of 
HIV and HBV co-infection in this study was found $12.56 \%$. This finding was closely the global situation in 2010 that was reported $10 \%$ of the global prevalence of hepatitis B virus (HBV) infection in HIV positive individuals (Soriano et al., 2010) (Klaus et al, 2015) that was also found $55.3 \%$ of co-infection with at least one of $\mathrm{HBV}, \mathrm{HCV}$, or syphilis. Moreover, $16.6 \%$ were infected with two of these agents, and $2.4 \%$ were positive for all three. Moreover, the prevalence of HBV co-infection in this study was higher than the study of Linda Aurpibul et al., which the prevalence of HBV/HIV co-infected was found $3.3 \%$ (95\% Cl: $1.9 \%$ to $5.2 \%$ ) and the protective antibody against HBV was found in $18 \%$ of population, and was significant higher among adolescents who received hepatitis $B$ revaccination after receiving antiretroviral therapy (93\% vs. $6 \%, \mathrm{p}<0.01)$ (Linda et al., 2012 ).

HIV and HBV Co-infection are common due to shared routes of transmission. In areas of low endemicity, such as North America, Australia and Europe, HBV and HIV infection are usually acquired in adulthood through sexual or percutaneous transmission. In that areas, the prevalence of chronic Co-infection is around 5-7\% among HIV-infected individuals (Alter, 2006). In countries with intermediate and high HBV endemicity, the main routes of transmission of HBV are prenatal or in early childhood; in these countries HBV co-infection rates are $10-20 \%$ (Lee, 2008; Nyirenda, 2008; Diop-Ndiaye, 2008).

$\mathrm{HIV} / \mathrm{HCV}$ co-infection in MSM. This study, the participants was the residents of Chiang Mai $(61.1 \%)$, Chiang Rai (22.2\%) and Phayao (16.7\%). The majority of this participants were Thai, Buddhism, 30-43 year old, employee , graduated secondary school, income 50025,500 baht per month, single, $10-18$ year old of $1^{\text {st }}$ sexual intercourse, had partner $\geq 1$ person, CD4>250, 1 10 year old of length of HIV Infection, 1-4 year old length of taking ARV Drug. Moreover, the high knowledge, high attitude and moderate practice were found $72.2 \%, 66.7 \%$ and $55.6 \%$ respectively.

The prevalence rate of $\mathrm{HIV}$ and $\mathrm{HCV}$ co-infection in this study was found $4.81 \%(18 / 374)$ that closely the study in Southern Brazil (Sonial, 2014) that was confirmed co-infection rate in MSM $3.51 \%$ of 93 HIVinfected patients who have attended a tertiary care academic hospital in Southern Brazil until 2014.The different of the associated factors of HCV and HIV coinfection between two studies were high frequency of drug use and lately investigations for the detection of coinfection. Moreover, Syphilis and viral infections, including HBV, HCV, HSV-2, CMV, and HR-HPV, were common in clinic based population of MSM in Toronto and more frequent among MSM living with HIV (Robert, 2016). The prevalence of HBV and/or HCV with HIV coinfection. In this study, the prevalence of HBV/HIV and $\mathrm{HCV} / \mathrm{HIV}$ in MSM were found $12.57 \%$ and $4.81 \%$ respectively, while the $\mathrm{HBV} / \mathrm{HCV} / \mathrm{HIV}$ co-intervention was only $0.28 \%$. Also, the significant associated factors of
HBV and/or HCV with HIV co-infection were age, smoking behavior, tongue piercing and Tenofivir drug taking. Nevertheless, Robert $S$ et al found age and number of lifetime male sexual partners were associated with HBV infection and lifetime injection drug use with HCV infection (Robert, 2016).

Fahimeh et al., found the prevalence of triple coinfections was very low and close to zero in the general population, health care workers and street children; while it peaked to $1.25 \%(95 \% \mathrm{Cl}: 0.00-3.01)$ in people who inject drugs (PWID). Patients who received multiple transfusions (PWRMT) and prisoners had a low prevalence as $0.01 \%$ and $0.28 \%$, not statistically different from the prevalence in the general population. Those finding were similarly this study which found $0.26 \%$ of triple co-infections in MSM group, but they found in people who inject drug (Fahimeh, 2016).

Jean Jacques et al., attempted to estimate the sera prevalence and identify risk factors associated with hepatitis $\mathrm{B}$ and/or $\mathrm{C}$ co-infections in HIV-infected individuals from five regions of Cameroon by screening $531 \mathrm{HIV}$ infected subjects for the presence of HBV surface antigen ( $\mathrm{HBsAg}$ ) and antibodies to HCV (HCV$\mathrm{Ab}$ ) in Iran. They found HBsAg but not HCV-Ab positivity was linked to age, lower CD4 count and residing in an urban rather than in a rural setting. Age was the same associated factor with co-infection when compare this study.(Jean, 2015)

Smoking behavior and tongue piercing were significant associated factors of $\mathrm{HBV}$ and/or HCV with HIV co-infection in this study that quite similar the Parviz et al., study that determined the prevalence of HBV, $\mathrm{HCV}$, and HIV infections among street children residing in southern Tehran (Parviz, 2009). They found smoking, tattooing, family history of hepatitis, drug abuse, imprisonment, sharing needles, urethral discharge, STD, and close contact with icteric cases significant difference $(P<0.05)$ between subjects with or without history of / existing HBV and HIV infection. The smoking with the other people in the same cigarette may likely infect both $\mathrm{HBV}$ and HCV when frequency exposure was occurred. They also found sixty percent of the participants had coinfection with HCV and HBV.

Dual infection with HBV and HIV was present in $76.9 \%$ of HIV positive subjects. All HIV-positive cases were also positive for HCV Ab. A total of 10 out of 202 participants (5\%) had triple infection with HIV, HBV, and HCV. A history of IDU had a strong association with HCV and HIV infections $(P<0.02)$ (Parvizal, 2009). On the other hand, Anouk et al., found that tattoo and/or piercing, like having a tattoo or piercing in an HBV endemic country, surface percentage tattooed, number of tattoos and piercings were not associated with either HBV or HCV (Anouk, 2011)

Tenofivir drug taking was the interesting significant associated factor of co-infection. The one reason that can explain this association was Tenofovir disoproxil fumarate 
can cause serious, life-threatening side effects. These include lactic acidosis (buildup of lactic acid in the blood) and severe liver problems such as the interactive between Tenofivir drug and Didanosine drug that was affect to Pancreatitis, Peripheral neuropathy. Therefore, if you have both HIV and hepatitis B virus (HBV) infection and take tenofovir, your HBV infection may get much worse (flare up) if you stop taking tenofovir. The HBV drug adefovir dipivoxil should not be taken with tenofovir. (AIDS info,2017)

\section{CONCLUSION}

The associated factors of HBV and/or HCV with HIV coinfection among MSM in the northern region, Thailand in 2015 were age, smoking behavior, tongue piercing and Tenofivir drug taking. Therefore, enhanced the surveillance and targeted prevention of viral hepatitis including improved sanitation and food safety, safer sex, efficiency healthcare delivery, disease prevention and vaccination coverage.

\section{ACKNOWLEDGMENTS}

The authors are grateful to the National Research Council of Thailand for the supporting grant (REH. 57005). The authors would like to thank the participants for their participation and also thank the all staff of the ARV clinics of 31 hospitals and all administration officer of Mae Fah Luang university, Phayao, Chiang Mai and Chiang Rai Public Health Provincial for all their cooperation and supporting.

\section{REFERENCES}

AIDS info (2017).Tenofovir Disoproxil Fumarate. Retrieved May 02 2017, from https://aidsinfo.nih.gov/drugs/290/tenofovir-disoproxilfumarate/0/patient

Alter MJ (2006). Epidemiology of viral hepatitis and HIV co infection. J Hepatol 2006;44(1 Suppl):S6-9.

Anouk TU, Anneke H, Albert B, Robin H, Lotte JB, Titia H, Roel AC, Maria P (2011). People with Multiple Tattoos and/or Piercings Are Not at Increased Risk for HBV or HCV in The Netherlands. PLoS ONE. September 2011 | Volume 6 | Issue 9 | e24736

Apidechkul T, Pongwiriyakul S (2016). Factors associated with HIV and HBV co-infection in Northern Thailand. Asian Pacific J. Tropical Disease. 6(3): 174-178

Centers for Disease Control and Prevention (2016,26 July). HIV/AIDS and Viral Hepatitis. Retrieved May 03, 2017, from https://www.cdc.gov/hepatitis/populations/hiv.htm

Centers for Disease Control and Prevention (2017, February).CDC FACT SHEET; HIV among Gay and Bisexual Men.Retrieved May 03, 2017, from https://www.cdc.gov/nchhstp/newsroom/docs/ factsheets/cdc-msm-508.pdf

Darawutmaprakorn N (2016). Estimate 3\% of Thai men are MSM, New information in Thailand. Retrieved May 03, 2017, from http://www.thairath.co.th/content/707729
Diop-Ndiaye H, Toure-Kane C,Hepatitis B (2008).seroprevalence and delta virus in HIV-1 Senegalese patients at HAART initiation (retrospective study). J Med Virol;80:1332-6.

Esteban Jl, van Helden J, Alborino F, Bürgisser $\mathrm{P}$, Cellerai $\mathrm{C}$ (2013).Multicenter evaluation of the elecsys $\Theta$ anti-HCV II assay for the diagnosis of hepatitis C virus infection. J. Med. Virol., 85: 13621368. doi: $10.1002 / j m v .23536$

Fahimeh BA, Ehsan M, Ali Mirzazadeh (2016). HIV, HBV and HCV Coinfection Prevalence in Iran - A Systematic Review and Meta Analysis. PLoS ONE 11(3): e0151946. doi:10.1371/journal. pone.0151946

Jean JN, Peter VA, Aubin JN, Lucy AA, Johnson NN, Phillipe N (2015). Hepatitis B and C Co-Infections in Some HIV Positive Populations in Cameroon, West Central Africa: Analysis of Samples Collected Over More Than a Decade. PLoS ONE 10 (9): e0137375. doi:10.1371/journal.pone.0137375

Klaus J, Michael T, Claus-Thomas B, Ramona S, Claudia K, Dieter M, Hans-Jochen H, Heiko J, Stephan D, Osamah H, Barra GB, Karolin(2015). High Prevalence and High Incidence of Coinfection with Hepatitis B, Hepatitis C, and Syphilis and Low Rate of Effective Vaccination against Hepatitis B in HIVPositive Men Who Have Sex with Men with Known Date of HIV Seroconversion in Germany. PLoS ONE. 10(11): 1-18

Lee HC, Ko NY, Lee NY (2008). Seroprevalence of viral hepatitis and sexually transmitted disease among adults with recently diagnosed HIV infection in southern Taiwan, 2000-5: upsurge in hepatitis C virus infections among injection drug users. J Formos Med Assoc 2008;107:404-11.

Linda A, Pagakrong L, Pope Kolasaraksa R (2012). HIV and Hepatitis B Co-infection Among Perinatally HIV-infected Tha Adolescents.Pediatr Infect Dis J. 31(9): 943-947

Muhlbacher A, Weber B, Burgisser $P$, Eiras A, Cabrera J, Louisirirotchanakul S, Tiller FW, Kim H.S, Helden J, Bossi V, Echevarria JM (2008). Multicenter study of a new fully automated HBsAg screening assay with enhanced sensitivity for the detection of HBV mutants.MedMicrobiollmmunol. Mar;197(1), 55-64.

Nyirenda M, Beadsworth MB, Stephany $P$ (2008).Prevalence of infection with hepatitis $B$ and $C$ virus and co infection with HIV in medical inpatients in Malawi. J Infect 2008;57:72-77.

Parviz V, Seyed MHM, Alireza F, Ramin MD (2009). Prevalence of HBV, HCV, HIV, and Syphilis among Homeless Subjects Older than Fifteen Years in Tehran. Arch Iranian Med 2009; 12 (5): 483 - 487

Soriano V, Vispo E, Labarga P, Medrano J, Barreiro P (2010).Viral hepatitis and HIV coinfection. Antivir. Res. 85, 303-315.

Sungkanuparph S, Vibhagool A, Manosuthi W, KiertiburanakulS, Atamasirikul K, Aumkhyan A, Thakkinstian A (2004). Prevalence of hepatitis $B$ virus and hepatitis $C$ virus co-infection with human immunodeficiency virus in Thai patients: a tertiary-care-based study. Retrieved May 03, 2017, from https://www.ncbi.nlm.nih.gov/ pubmed/15825712

Robert SR, Juan L, Mona R. Loutfy, Wangari T, Anuradha R, Sanja H, Maya K, Roberta H, Troy G, Jason B, Graham S, Tatjana R, Rupert K (2016). Prevalence of Sexually Transmitted Viral and Bacterial Infections in HIV-Positive and HIV Negative Men Who Have Sex with Men in Toronto. PLoS ONE 11(7): e0158090. doi:10.1371/journal.pone.0158090

Sonia MR, Felipe FT, Nayara PB, Henrique D, Andre O, Denis L, Andressa GS, Bárbara PL, João PF, Maria LP (2014). Human immunodeficiency virus and hepatitis $C$ virus/hepatitis $B$ virus coinfection in Southern Brazil: clinical and epidemiological evaluation. The Brazilian J. INFECTIOUS DISEASES.2014; 1 8(6):664-668

World Health Organization (2016, November). 10 facts on HIV/AIDS. Retrieved May 03, 2017, from http://www.who.int/features /factfiles/hiv/en/

World Health Organization (2017).HIV and hepatitis. Retrieved May 03 , 2017, from http://www.who.int/hiv/topics/hepatitis/hepatitisinfo 